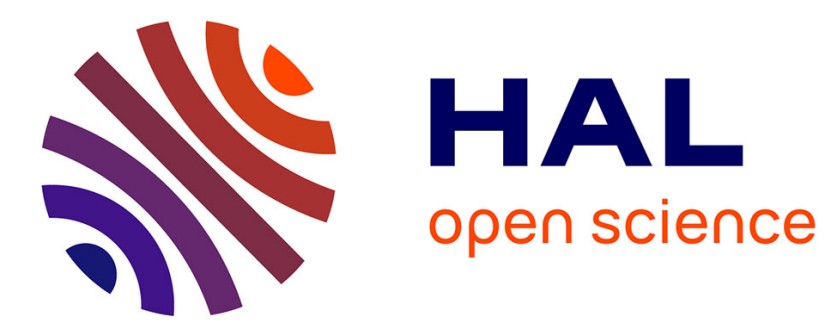

\title{
Simulation of Viscous Fingering Instability by the Lattice Boltzmann Method
}

Lucien Vienne, Simon Marie, Francesco Grasso

\section{To cite this version:}

Lucien Vienne, Simon Marie, Francesco Grasso. Simulation of Viscous Fingering Instability by the Lattice Boltzmann Method. AIAA Aviation 2019 Forum, Jun 2019, Dallas, United States. pp.1-13, 10.2514/6.2019-3432 . hal-03091058

\section{HAL Id: hal-03091058 https://hal.science/hal-03091058}

Submitted on 30 Dec 2020

HAL is a multi-disciplinary open access archive for the deposit and dissemination of scientific research documents, whether they are published or not. The documents may come from teaching and research institutions in France or abroad, or from public or private research centers.
L'archive ouverte pluridisciplinaire HAL, est destinée au dépôt et à la diffusion de documents scientifiques de niveau recherche, publiés ou non, émanant des établissements d'enseignement et de recherche français ou étrangers, des laboratoires publics ou privés. 


\title{
Simulation of Viscous Fingering Instability by the Lattice Boltzmann Method
}

\author{
Lucien Vienne*, Simon Marié ${ }^{\dagger}$, and Francesco Grasso \\ DynFluid laboratory, 151 boulevard de l'hôpital, 75013 Paris, France
}

\begin{abstract}
The viscous fingering instability is successfully simulated within a lattice Boltzmann framework. Each species of the mixture is governed by its own kinetic equation and a force takes into account the diffusion between species. The influence of the porous medium is mimicked by using the gray lattice Boltzmann model or the Brinkman force model. In this study, both representations of the porous medium yield equivalent results. Then a physical analysis of the instability is performed and two different dynamical behaviour are stated and discussed. Finally, it is observed that a high Péclet number intensify the instability and the viscous dissipation stemming from the Darcy-Brinkman equations delay the development of the fingers in the case of large effective viscosity.
\end{abstract}

\section{Introduction}

Mixing in porous media is particularly difficult due to the absence of inertia but it plays a key role in carbon sequestration, oil recovery, mantle convection, microfluidic device, and chromatographic columns. High Reynolds number flows can be actively stirred by turbulence but other mechanisms are needed in a porous medium. The generation of an interfacial instability is one mechanism among others that can improve the mixing. This instability can be driven by chemical reactions, density stratification (Rayleigh-Taylor instability), or difference in viscosity. Here, we focus on the latter, which is called viscous fingering or Saffman-Taylor instability. This interfacial instability occurs when a less viscous fluid displaces a more viscous fluid in a porous medium [1]. Finger-like patterns emerge and grow, exhibiting complex dynamics.

Since the pioneer work of Hill [2] in 1952 and Saffman and Taylor [3] in 1958, extensive investigations has been performed on both immiscible and miscible fluids. A distinctive characteristic of miscible fluids is the lack of a sharp interface. Mixing produces changes to the fluid since the properties of the mixture vary continuously while diffusion occurs. Usually, the viscous fingering is modeled by using Darcy's law and a scalar transport equation for the concentration. A relation between the viscosity and the concentration of the components must be defined. Most articles follow the standard literature rather than a physical rigor and assume an exponential dependence. In this paper, the viscous fingering instability is simulated with a lattice Boltzmann framework. The interactions between the species of the mixtures are taken into account by means of a forcing term. This model for miscible fluids has been recently introduced in Ref. [4]. One of the important feature of this model lies in the computation of the viscosity coefficient which has a more rigorous physical foundation, stemming from approximations of the kinetic theory of gases extended to mixtures. Adopting a lattice Boltzmann approach, we investigate two strategies so as to mimic the porous medium effects: the gray lattice Boltzmann model assuming a partial reflection condition and the Brinkman force model adding a drag force.

This paper is laid out as follows. In the first section, we present the lattice Boltzmann model for miscible mixture, and how the porous medium is modeled. In the second section, the viscous fingering dynamic is highlighted. The development of the instability is studied globally through the evolution of the mixing length. Then, we focus on the two main stages of the instability, the first one in the early times when the fingers grow linearly and the second one when the fingers interact with strong non-linearity. The influence of different parameters such as the ratio of the rate of advection to the rate of diffusion (Péclet number), the porous representation, and the effective viscosity are underlined.

\footnotetext{
*Ph.D. student, Conservatoire National des Arts et Métiers, contact@lvienne.com

$\dagger^{\dagger}$ Assistant professor, Conservatoire National des Arts et Métiers, simon.marie@lecnam.net

† Professor, Conservatoire National des Arts et Métiers, francesco.grasso@lecnam.net
} 


\section{Model}

\section{A. Simplified kinetic model for miscible mixtures}

The lattice Boltzmann method is an alternative method for simulating fluid flows by solving a simplified formulation of the kinetic model. A mixture is composed of multiple species and each species is defined by its own distribution function, which is governed by its own kinetic equation. The present lattice Boltzmann model for miscible mixtures is based on a forcing term approach. We summarize here its main features and a more detailed presentation can be found in Ref. [4].

Let $m$ and $n$ denote different species ( $m, n=1,2, \ldots, N ; N$ being the total number of species). The distribution function of species $m, f_{\alpha}^{m}$, obeys the following discrete kinetic equation

$$
f_{\alpha}^{m}\left(\mathbf{x}+\mathbf{e}_{\alpha} \delta_{t}, t+\delta_{t}\right)=f_{\alpha}^{m}(\mathbf{x}, t)-\frac{\delta_{t}}{\tau_{m}}\left[f_{\alpha}^{m}(\mathbf{x}, t)-f_{\alpha}^{m(e q)}(\mathbf{x}, t)\right]+\delta_{t} S_{\alpha}^{m}(\mathbf{x}, t)
$$

where $\mathbf{x}, t, \alpha$, and $\tau_{m}$ are, respectively, the spatial coordinate, the time, the number of discrete kinetic velocities $\mathbf{e}_{\alpha}$, and the relaxation time of each species. Usually, the left and right hand sides of Eq. (1) are processed separately into two steps: streaming and collision, respectively. The equilibrium distribution functions, $f_{\alpha}^{m(e q)}$, are given by the standard polynomial formulation,

$$
f_{\alpha}^{m(e q)}=\rho_{m} \omega_{\alpha}\left[1+\frac{\mathbf{u}_{m} \cdot \mathbf{e}_{\alpha}}{c_{s}^{2}}+\frac{\left(\mathbf{u}_{m} \cdot \mathbf{e}_{\alpha}\right)^{2}}{2 c_{s}^{4}}-\frac{\mathbf{u}_{m} \cdot \mathbf{u}_{m}}{2 c_{s}^{2}}\right] .
$$

$S_{\alpha}^{m}$ is the source term from Guo's forcing scheme [5], widely used in order to include forces in the lattice Boltzmann algorithm,

$$
S_{\alpha}^{m}=\left(1-\frac{\delta_{t}}{2 \tau_{m}}\right) \omega_{\alpha}\left[\frac{\mathbf{e}_{\alpha}-\mathbf{u}_{m}}{c_{s}^{2}}+\frac{\left(\mathbf{e}_{\alpha} \cdot \mathbf{u}_{m}\right) \mathbf{e}_{\alpha}}{c_{s}^{4}}\right] \cdot \mathcal{F}_{m}
$$

$\mathcal{F}_{m}$ being the force acting on the $m$-th species which is derived in the following to take into account the species interactions.

In this study, we use the so-called D2Q9 isothermal, two-dimensional and nine-velocity discretisation. Extension to the three-dimensional formulation (D3Q19 or D3Q27) is straightforward. The pseudo-sound velocity is $c_{s}^{2}=\frac{1}{3}$, the kinetic velocities are expressed as

$$
\mathbf{e}_{\alpha}=\left[\begin{array}{ccccccccc}
0 & 1 & 0 & -1 & 0 & 1 & -1 & -1 & 1 \\
0 & 0 & 1 & 0 & -1 & 1 & 1 & -1 & -1
\end{array}\right]^{T} \quad 1 \leq \alpha \leq 9
$$

and the lattice weights are equals to

$$
\omega_{\alpha}=\left[\begin{array}{lllllllll}
\frac{4}{9} & \frac{1}{9} & \frac{1}{9} & \frac{1}{9} & \frac{1}{9} & \frac{1}{36} & \frac{1}{36} & \frac{1}{36} & \frac{1}{36}
\end{array}\right]^{T} \quad 1 \leq \alpha \leq 9 .
$$

The macroscopic quantities, namely the density and momentum of each species, are obtained by computing the different moments of the distribution functions.

$$
\rho_{m}=\sum_{\alpha} f_{\alpha}^{m}, \quad \rho_{m} \mathbf{u}_{m}=\sum_{\alpha} f_{\alpha}^{m} \mathbf{e}_{\alpha}+\frac{\delta_{t}}{2} \mathcal{F}_{m}
$$

The resulting macroscopic equations are the conservation equations for low Mach number flows subjected to a body force (e.g. gravity). In order to take into account the interaction of miscible species, we introduce diffusion forces. These forces are derived from kinetic theory by Kerkhof and Geboers [6],

$$
\mathcal{F}_{D, m}=-p \sum_{n=1}^{N} \frac{x_{m} x_{n}}{\mathcal{D}_{m n}}\left(\mathbf{u}_{m}-\mathbf{u}_{n}\right),
$$

and $\mathcal{F}_{m}$ becomes

$$
\mathcal{F}_{m}=\mathcal{F}_{D, m}+\mathcal{F}_{B, m}
$$

where $\mathcal{F}_{B, m}$ is a body force and $p$ is the total pressure given by Dalton's law $p=\sum_{m} p_{m}$. As a result, the discrete kinetic equations Eq. (1) for the various species are coupled through $\mathcal{F}_{m}$. Since the diffusion force $\mathcal{F}_{D, m}$ depends on 
the velocity, total pressure $p$, molar fractions $x_{m}$, and (Maxwell-Stefan or binary) diffusion coefficients $\mathcal{D}_{m n}$, a linear system must be solved at each time step in order to compute the species momentum by means of Eq. (6).

In the standard lattice Boltzmann equation, the pseudo (isothermal) speed of sound $\left(c_{s}\right)$ is fixed by the lattice. For D2Q9 velocity set, $c_{s}$ is equal to $c_{s}^{2}=1 / 3$ for all species which is not the case for mixture of species having different molecular masses. Recalling that the partial pressure obeys the ideal gas law, $p_{m}=\rho_{m} c_{s, m}^{2}$ and $c_{s, m}^{2}=R T / M_{m}$, where $R$ is the universal gas constant, $T$ is the temperature, and $M_{m}$ is the species molecular mass; we introduce the following body force in order to take into account species having different molecular masses

$$
\mathcal{F}_{B, m}=\left(1-\beta_{m}\right) c_{s}^{2} \nabla \rho_{m},
$$

where the gradient term is computed by means of a compact scheme

$$
\nabla \rho_{m}(\mathbf{x})=\frac{1}{c_{s}^{2}} \sum_{\alpha} \omega_{\alpha} \mathbf{e}_{\alpha} \rho_{m}\left(\mathbf{x}+\mathbf{e}_{\alpha}\right) .
$$

The partial pressure then becomes

$$
p_{m}=\beta_{m} c_{s}^{2} \rho_{m}
$$

where $\beta_{m}=c_{s, m}^{2} / c_{s}^{2}$ is the ratio between the species and the standard LBM pseudo speeds of sound.

The lattice Boltzmann scheme still retains a connection with the macroscopic scale through the moments of the distribution functions (Eqs. 6, and the relation between the relaxation time and the viscosity stemming from the Chapman-Enskog expansion (see V.A

$$
\mu_{m}=\rho_{m} c_{s}^{2}\left(\tau_{m}-\frac{\delta_{t}}{2}\right)
$$

The viscosity of a species has a similar formulation as Wilke's law [4]

$$
\mu_{m}=\frac{x_{m} \mu_{0, m}}{\sum_{n}^{N} x_{n} \Phi_{m n}}
$$

where $\mu_{0, m}$ is the viscosity of the pure species $m, x_{m}$ is the molar fraction of species $m$, and

$$
\Phi_{m n}=\frac{1}{2 \sqrt{2}}\left(1+\frac{M_{m}}{M_{n}}\right)^{-\frac{1}{2}}\left[1+\left(\frac{\mu_{0, m}}{\mu_{0, n}}\right)^{\frac{1}{2}}\left(\frac{M_{n}}{M_{m}}\right)^{\frac{1}{4}}\right]^{2}
$$

Complex diffusion between species can successfully be simulated by the present approach [4]. For purely diffusive flow, the dynamic predicted by Maxwell-Stefan's equations is recovered and non-Fickian behaviors such as reverse, osmotic or barrier diffusion may occur.

\section{B. Porous medium}

Porous media, such as natural soils or sedimentary rocks, are incredibly difficult to model due to the heterogeneous structure and composition of the pores at many different scales. Moreover, the simulation of a large-scale flow at the pore scale is often impractical because of long CPU time and excessive memory consumption. Hence, a volume average approach is usually used to overcome these difficulties. In this case, Darcy or Darcy-Brinkman semi-empirical equations are solved and porosity, permeability, and other measurements are defined. In the case of a single fluid, the Brinkman equation adds a drag force to the Stokes equations,

$$
\nabla p=-\frac{\mu}{K} \mathbf{u}+\nabla \cdot\left[\mu_{e}\left(\nabla \mathbf{u}+(\nabla \mathbf{u})^{T}\right)\right]
$$

where $p$ is the pressure, $K$ is the permeability of the medium, $\mathbf{u}$ is the fluid velocity, and $\mu_{e}$ is the effective viscosity which may not be equal the fluid viscosity. The Brinkman equations are a convenient transition model between Darcy regime $\left(K \rightarrow 0\right.$ or $\left.\nabla \cdot\left[\mu_{e}\left(\nabla \mathbf{u}+(\nabla \mathbf{u})^{T}\right)\right] \rightarrow 0\right)$ and Stokes regimes $(K \rightarrow+\infty)$.

It is to be noted that the dynamics of the viscous fingering is mainly governed by the Darcy equation [1] thereby the Brinkman term, $\nabla \cdot\left[\mu_{e}\left(\nabla \mathbf{u}+(\nabla \mathbf{u})^{T}\right)\right]$, is not of primary importance and the effective viscosity is taken equal to the fluid viscosity unless otherwise stated. Indeed, the literature [1, 7-10] only considers Darcy's equations. The latter equations correctly described flows in Hele-Shaw cells and in simple porous media whereas Darcy-Brinkman's 
equations are often used as a transition model between porous flow and Stokes flow such as in the case of fractured porous media. In order to give some insight on the influence of the effective viscosity, three simulations are also performed with different ratios of viscosity and effective viscosity .

Furthermore, the porous medium is assumed to be isotropic and the permeability is given by a scalar value. Viscous fingering in anisotropic media is studied in Ref. [11]. The originality of this work is the use of the aforementioned lattice Boltzmann model for miscible mixtures. Neither transport equation for the concentration, nor Darcy equation with exponential viscosity dependency are solved. Each species dynamic is governed by its own kinetic equation (Eq. (1)) and the viscosity of species stemmed from the kinetic theory of gases (Eq. (13)). In this section, we present how the porous medium effects are introduced in the lattice Boltzmann framework.

\section{Gray lattice Boltzmann}

Taking the advantage of the mesoscopic view of the lattice Boltzmann method, a porous medium can be modeled by different means. We first present the so-called gray lattice Boltzmann scheme where a special condition is applied on the whole domain: in each cell during the streaming step, the distribution functions are bounced back with a certain amount. Various schemes are proposed in the literature. In Ref. [12], the local pre-collision distribution functions are reflected whereas in Ref. [13], the local post-collision distribution functions are taken as reflected distribution functions. Yoshida and Hayashi [14] also suggest a gray lattice Boltzmann model based on Ref. [15] but the neighboring post-collision distribution functions are reflected. Despite some differences, all the previous gray lattice Boltzmann schemes [12-14] recover the Darcy-Brinkman equation [16]. In this study, the use of the model proposed in Ref. [14] is motivated by two main reasons. First, a redefinition of the macroscopic velocity is not required in this latter scheme. This redefinition would lead to complicated expressions especially on the boundary nodes since in the present approach, velocity and forces are coupled (Eqs. (6)77). Furthermore, the classical bounce-back boundary condition [17] is obtained as a limit expression. If we use the notation $\Gamma_{\alpha}^{m}$ to substitute for the right-hand side of Eq. (1) (post-collision part), the following gray lattice Boltzmann scheme is obtained,

$$
f_{\alpha}^{m}\left(\mathbf{x}+\mathbf{e}_{\alpha} \delta_{t}, t+\delta_{t}\right)=\left(1-\theta_{m}\right) \Gamma_{\alpha}^{m}(\mathbf{x}, t)+\theta_{m} \Gamma_{\bar{\alpha}}^{m}\left(\mathbf{x}+\mathbf{e}_{\alpha} \delta_{t}, t\right),
$$

where $\Gamma_{\bar{\alpha}}^{m}\left(\mathbf{x}+\mathbf{e}_{\alpha} \delta_{t}, t\right)$ are the post-collision distribution functions from the opposite direction of $\alpha$ velocity located at the neighboring node and $\theta_{m}$ is the amount of reflection. When $\theta_{m}=0$, the usual streaming step occurs and $\theta_{m}=1$ is similar to the classical bounce-back (no-slip) condition [17]. For $0<\theta_{m}<1$, the scheme mimics the dynamics of a porous medium and this approach should be related to a subgrid model for porous medium. The parameter $\theta_{m}$ depends on the viscosity of the fluid and the permeability of the considered porous medium and the following relation can be obtained [14, 16]

$$
\frac{\mu_{m}}{K \rho_{m}}=\frac{2 \theta_{m}}{\left(1-\theta_{m}\right) \delta_{t}}
$$

Here, $\theta$ is defined on the link between nodes instead of being a node based value. In each direction $\alpha, \theta$ is computed by the means of an interpolation between the two nodes. This ensures that the mass is conserved strictly by the scheme.

\section{Brinkman drag force}

In the second approach, referred as Brinkman force model, the porous medium resistance is included explicitly through a drag force to the lattice Boltzmann equation, e.g. [18-21]. Here, we follow a similar approach as in Ref. [22] and the forcing term is written as

$$
\mathcal{F}_{\text {porous }, m}=-\frac{\mu_{m}}{K} \mathbf{u}_{m}
$$

As mentioned in Refs. [23-25], the numerical permeability depends lightly on the viscosity for both Brinkman force and gray lattice Boltzmann models. This nonphysical variation can be removed by keeping the so-called magic number $\Lambda$ constant (see [18, 23--25]). We use the multiple relaxation times (MRT) collision model [26] with $\Lambda=3 / 16$ corresponding to $s_{q}=8 \frac{2-1 / \tau_{m}}{8-1 / \tau_{m}}[17,26]$. 


\section{Results and discussions}

\section{A. Darcy law}

In order to show that Darcy's law is recovered by both of the proposed schemes, a flow composed of two identical species $\left(m=1,2\right.$ and $\left.\beta_{m}=1\right)$ through a homogeneous porous medium is simulated. Domain size is set to $\left(n_{x}=101, n_{y}=20\right)$. Periodic conditions are applied on top and bottom of the domain and a constant velocity profile $\left(u_{x, m}=U, u_{y, m}=0\right)$ is imposed at left and right side of the domain [27]. Distribution functions are initialized at their equilibrium values $f_{\alpha}^{m}(t=0)=f_{\alpha}^{m(e q)}\left(\rho_{m}, u_{x, m}=U, u_{y, m}=0\right)$ with an uniform initial density field $\rho_{1}=\rho_{2}$. Simulation is stopped when steady state is achieved. In Fig 1 the pressure drop between the left and right side of the domain is plotted against the horizontal inlet velocity. Both gray lattice Boltzmann and Brinkman force models give equivalent results and show the expected Darcy behavior of the simulated system.

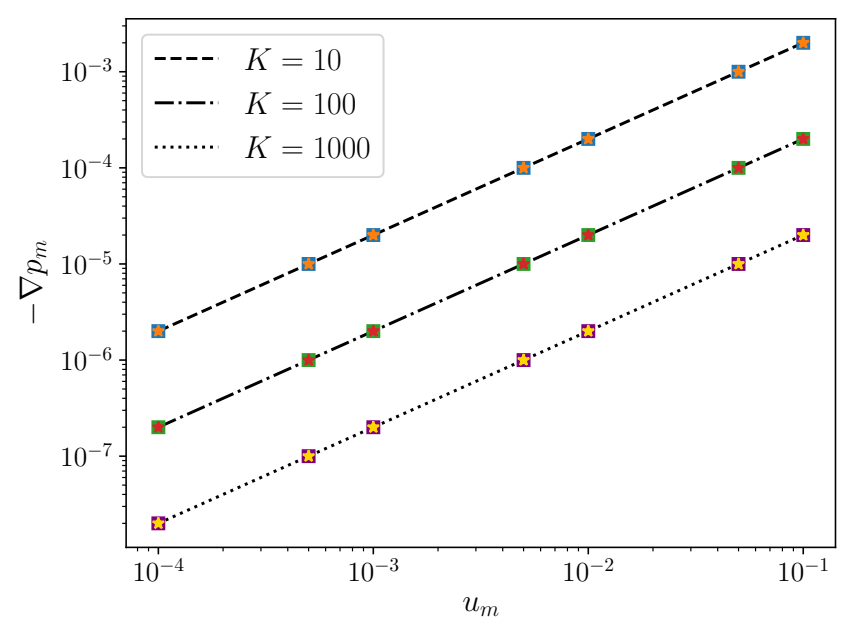

Fig. 1 Pressure drop according to the velocity for different permeability at fixed viscosity $\mu_{m}=2 \times 10^{-1}$. Values are given in lattice units. Squares and stars stand for numerical results obtained respectively with the gray lattice Boltzmann and the Brinkman force schemes. Lines are the analytical solution $-\nabla p_{m}=\mu_{m} / K U$.

This first result shows that the present model is able to accurately reproduce the standard behaviour of a porous medium for different values of $K$ and for both porous modeling strategies.

\section{B. Viscous fingering}

\section{Global description of the instability}

The viscous fingering instability occurs when a less viscous fluid invade a more viscous fluid. In the case of miscible fluids, if the interface between fluids is sharp enough, finger-like patterns emerge and grow, exhibiting complex dynamics.

In the following simulations, the fluid consists of two species having equal molar masses $\left(m=1,2 \beta_{m}=1\right)$. The resting mixture is composed of molar fractions $x_{1}=0.1$ and $x_{2}=0.9$ and these are swapped for the injected mixture $\left(x_{1}=0.9, x_{2}=0.1\right)$. Following the literature, dimensionless parameters are defined such as the log-viscosity ratio and the Péclet number (ratio of the advective transport rate and the diffusive transport rate)

$$
R=\ln \left(\frac{\mu_{0,2}}{\mu_{0,1}}\right), \quad P_{e}=\frac{U L_{r e f}}{\mathcal{D}_{12}}
$$

where $U$ is the injected velocity and $L_{r e f}$ is the reference length. Here, the characteristic scales are based on the convective length $L_{r e f}=n_{y}$ and time $t_{r e f}=n_{y} / U$ with $n_{y}$ the height of the domain. If $R<0$, the invading fluid is more viscous than the resting fluid, and the interface is always stable. On the other hand, if $R>0$, the invading fluid is less viscous than the resting fluid, and the interface between the two fluids may be unstable. The number of fingers increases with both the Péclet number and the ratio $R$. We recall that the relation between the pure viscosity and the 
species is given by Eq. (13). The influence of different parameters on the instability is studied. First, we compare the results obtained from the gray lattice Boltzmann and the Brinkman force models. Afterward, the effects of the Péclet number on the viscous fingering are highlighted. Finally, we present the differences on the emergence of the fingers caused by the effective viscosity.

The domain considered is two-dimensional, $\left(n_{x}=1024, n_{y}=512\right)$ unless otherwise specified, and we impose periodicity at the top and bottom boundaries. At the left and right boundaries, a constant velocity condition equal to $U$ is applied for both species. In order to compare our results, we keep the reference pressure constant $p_{\text {ref }}=\mu_{0,1} U n_{y} / K=1$ and the permeability of the porous medium is set to $K=1$. Each simulation is initialized by an almost sharp interface with a small perturbation so as to trigger the instability,

$$
\begin{aligned}
& x_{1}(x, y, t=0)=0.9+(0.9-0.1)\left[0.5+0.5 \times \operatorname{erf}\left(\frac{x-50}{\sqrt{t_{0}}}\right)+r(y) \times 10^{-3} \exp \left(\frac{-(x-50)^{2}}{\sqrt{t_{0}}}\right)\right], \\
& x_{2}(x, y, t=0)=1-x_{1}(x, y, t=0),
\end{aligned}
$$

where the function $r$ returns a random number which is uniformly distributed in the interval $[-1,1]$. In each simulation, the same seed is used for the pseudo-random generator. We set $t_{0}=10^{-6}$ to avoid strong gradients. The initial total pressure is computed from Darcy's law for the mixture

$$
p(x, y, t=0)=p_{r e f}+\left(1-\frac{x}{n_{x}}\right) \frac{\mu_{1}+\mu_{2}}{K} U
$$

and the distribution functions are initialized at their equilibrium values $f_{\alpha}^{m}(t=0)=f_{\alpha}^{m(e q)}\left(\rho_{m}, u_{x, m}=U, u_{y, m}=0\right)$.

As the instability evolves, fingers develop first linearly then interact non-linearly, and the dynamics become chaotic. Before exploring theses different regimes, we examine the global dynamics of the viscous fingering. The average molar fraction over the transverse direction is a classical measurement

$$
\bar{x}(x, t)=\frac{1}{n_{y}} \int x_{1}(x, y, t) d y,
$$

as well as the length of the mixing zone, which is defined here as

$$
l_{\text {mix }}(t)=\frac{1}{L_{\text {ref }}}\left(\left.x\right|_{\bar{x}(x, t)=0.9-10^{-3}}-\left.x\right|_{\bar{x}(x, t)=0.1+10^{-3}}\right)
$$

In Figure 2, the mixing length is represented in logarithmic scale according to the non-dimensional time $t^{*}=t / t_{r e f}$ for various Péclet number at $R=3$. Two different regimes are clearly visible. In the first regime up to $t^{*}=0.13$, the diffusion dominates the growth of the mixing length, which is proportional to $\sqrt{t^{*}}$ as in the case of no viscosity contrast $(R=0)$. Afterward, a transition regime occurs and depends on the Péclet number. The second regime can be seen in Fig. 3 The mixing length is represented in linear scale to better highlight the longtime behavior and the linear evolution of the fingers. The growth of the mixing length is linear in time suggesting that the advection now dominates. It is interesting to note that both regimes exist even when the initial molar fractions are not perturbed and no finger emerges. In this case, the development of the mixing length is reduced in the second regime. For instance in Fig. 3, the approximate slope is 1 with no finger, whereas the slope is around 2.2 with fingering. Compared to the pure diffusive case, the mixing length is heavily affected by the viscous fingering instability. Moreover, Fig. 3 shows no significant difference between the gray lattice Boltzmann and the Brinkman force model. The small deviation for $t^{*}>6.5$ is probably due to the influence of the boundary condition close to the location of the fingers. Same dynamic results independently of how the porous media is modeled confirming that the gray lattice Boltzmann or the Brinkman force adequately reproduce the viscous fingering instability.

The development of the instability is shown in Fig. 4. The onset of fingers is observed on the diffusive regime. Then, the fingers interact via highly non-linear mechanisms such as merging, splitting, etc (more details are given in section III.B.3 during the transition between diffusive and advective regimes $\left(0.13<t^{*}<3\right)$. A better measurement of the transition between linear and non-linear behavior is given by a sudden growth of the interfacial length $I(t)=\frac{1}{L_{\text {ref }}} \iint\left[\left(\partial x_{1} / \partial x\right)^{2}+\left(\partial x_{1} / \partial y\right)^{2}\right]^{\frac{1}{2}} d x d y$. Finally for $t^{*}>3$, the few remaining fingers grows at constant rate. At very late time (not observed here), the fingers should mix with the ambient fluid and are convected at a given speed close to the injected velocity. The length of the fingers should stay roughly the same since the interface is diffuse enough to smooth out the viscosity and molar gradients. 


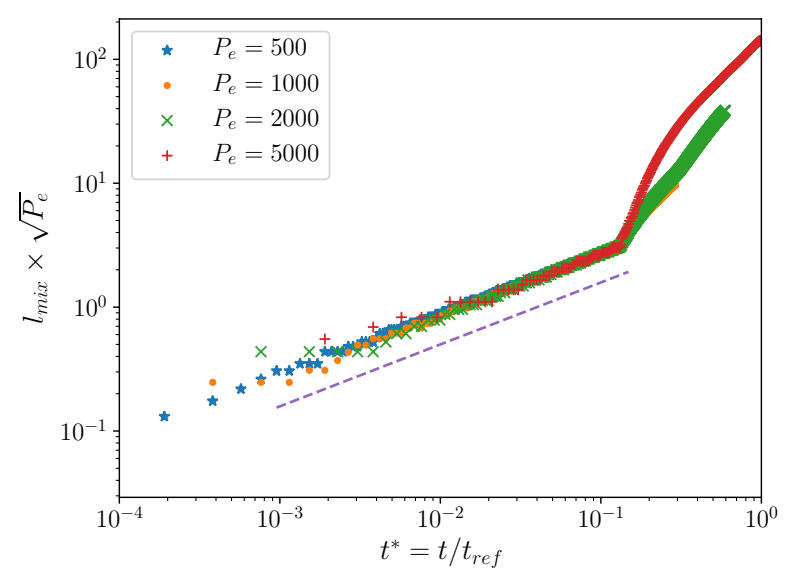

Fig. 2 Mixing length multiplied by $\sqrt{P e}$ at various Péclet for $R=3$ with the gray lattice Boltzmann model. Dashed line shows a power law with exponent $1 / 2$.

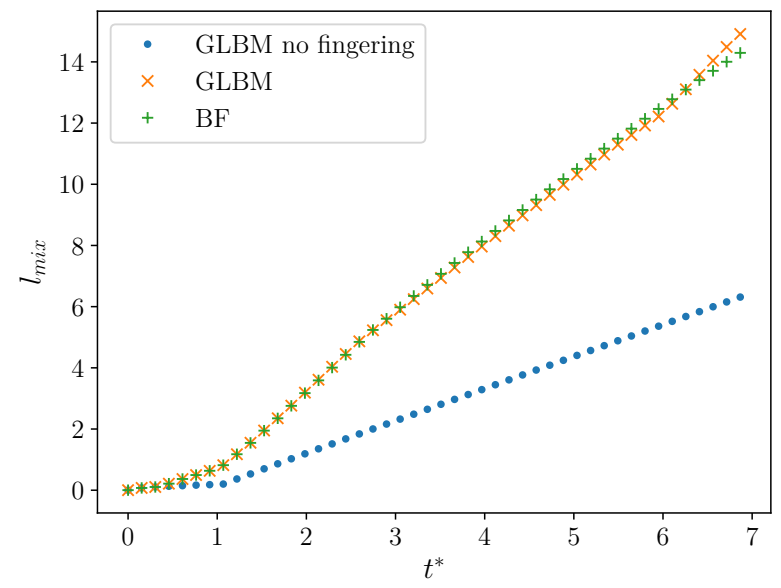

Fig. 3 Mixing length for $R=3, P e=2000$. Simulations are performed with the gray lattice Boltzmann and Brinkman force models on a larger domain $\left(n_{x}=8192, n_{y}=512\right)$. Blue dots stand for a simulation carried out with no initial perturbation.

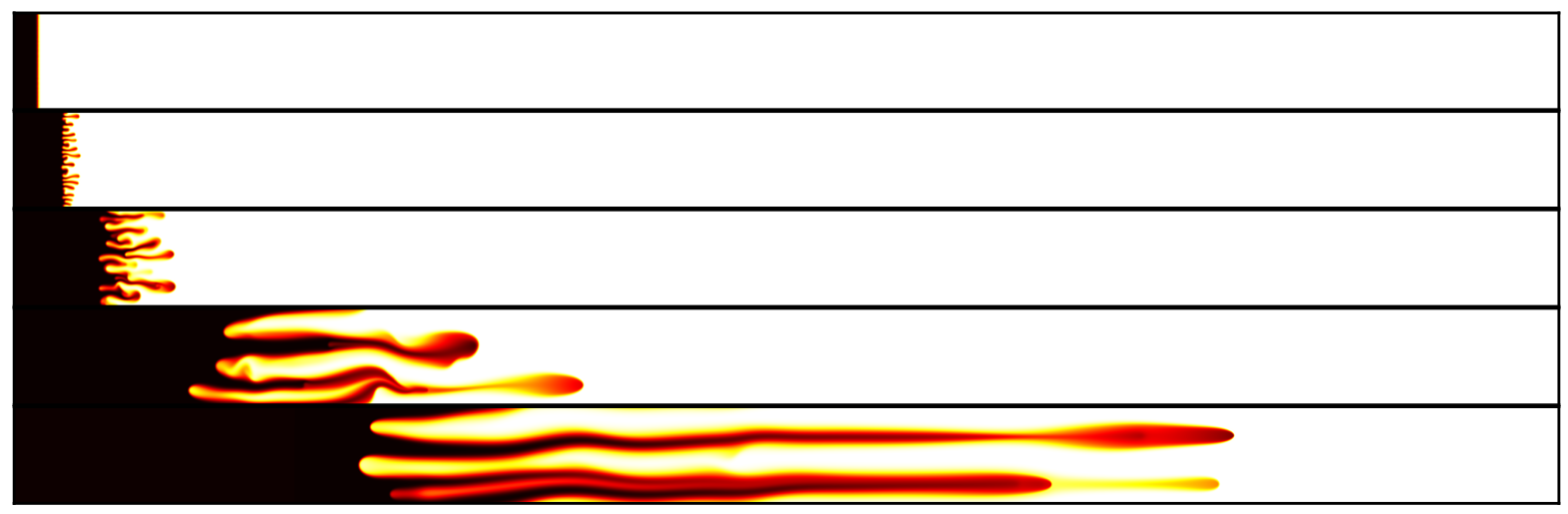

Fig. 4 Instability development for $R=3, P_{e}=2000$ at $t^{*} \approx 0.15,0.46,1.07,3.05,6.10$. The whole domain $\left(n_{x}=8192, n_{y}=512\right)$ is plotted. The color-map (black-red-yellow-white) represents the variation of the molar fraction from $x_{1}=0.9$ to $x_{1}=0.1$. Same scaling is used for both $\mathbf{x}$ and $\mathbf{y}$-axis.

In order to give some insight on the influence of the effective viscosity, three simulations are performed with $\mu_{e, m}=0.1 \mu_{m}, \mu_{e, m}=\mu_{m}, \mu_{e, m}=10 \mu_{m}$. As expected, the diffusion caused by the effective viscosity affects the instability as shown in Fig. 5. The development of fingers is delayed in the case of a high effective viscosity. The dynamic when no finger emerges does not dependent on the effective viscosity. The influence of the fingers on the mixing length becomes only apparent at $t^{*} \approx 0.6$ for $\mu_{e, m}=10 \mu_{m}$ instead of $t^{*} \approx 0.3$ for $\mu_{e, m}=\mu_{m}$. In contrast, the differences between $\mu_{e, m}=0.1 \mu_{m}$ and $\mu_{e, m}=\mu_{m}$ are small. Thus, when the effective viscosity is sufficiently small, the Darcy drag is the dominant term in Eq. (15) and the development of fingers is not altered.

In this section, the global development of the instability has been discussed. We now focus on two specific parts of the viscous fingering, namely the very beginning of the onset of the instability when the fingers grow linearly and the intermediate times when strong non-linear interactions occur. 


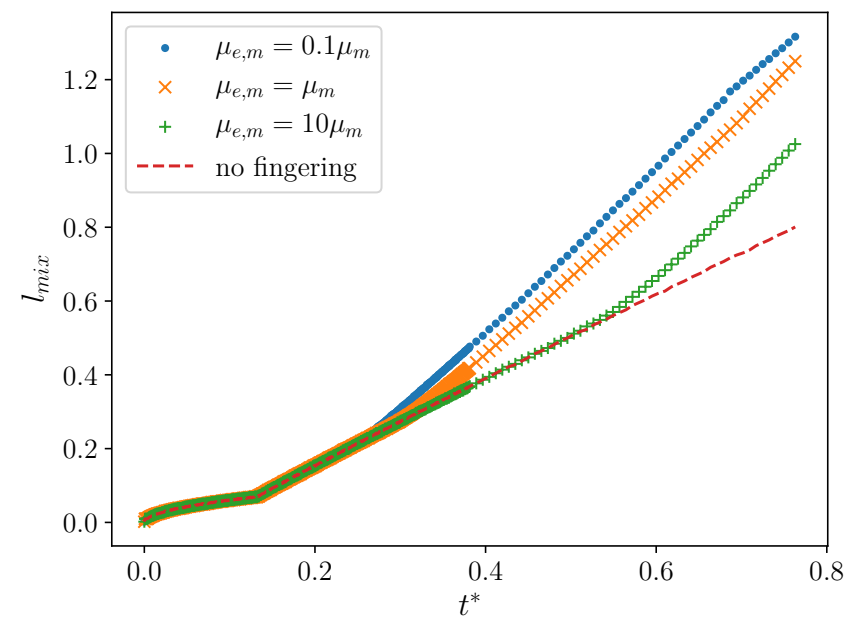

Fig. 5 Mixing length for different values of the effective viscosity and $R=3, P_{e}=2000$ with the gray lattice Boltzmann model.

\section{Early times: growth rate of the perturbation}

At early times, the interface between the two mixtures spread because of diffusion and fingers develop. At the very beginning, the flow is linearly unstable and perturbations grow exponentially. The onset of fingers is explored by many authors using linear stability analysis [1]. Because of diffusion, the base flow is unsteady. Tan and Homsy [7] use a quasi-steady state approximation in which the base flow is frozen and modal analysis is performed at successive frozen time. This method is commonly used to study the viscous fingering [8, 11]. Non modal analysis is carried out in Ref.[9] and shows some discrepancy with linear stability analysis.

Linear stability analysis can also be directly performed in the lattice Boltzmann framework (see. [28, 29]) however this approach is still in its infancy and is only applied to simple single fluid flow. In this study we carry out non linear simulation with the aforementioned lattice Boltzmann model and compute the growth rate of the perturbations. The molar fractions can be decomposed into a base state $x_{m}^{0}$ and a perturbative component $x_{m}^{\prime}$,

$$
x_{m}(\mathbf{x}, t)=x_{m}^{0}(\mathbf{x}, t)+x_{m}^{\prime}(\mathbf{x}, t) .
$$

If we assume that the perturbation can be expressed in the form of

$$
x_{m}^{\prime}(\mathbf{x}, t)=x_{m}^{\prime}(\mathbf{x}) \exp (\sigma t)
$$

where $\sigma$ is the growth rate of the perturbation. The dispersion curves are obtained by applying a fast Fourier transform (FFT),

$$
\begin{aligned}
x_{m}^{\prime}(\mathbf{x}, t) & =x_{m}^{0}(\mathbf{x}, t)-x_{m}(\mathbf{x}, t) \\
\hat{x}(x, k, t) & =\operatorname{FFT}_{y}\left(x_{m}^{\prime}(\mathbf{x}, t)\right) \\
a(k, t) & =\|\hat{x}(x, k, t)\|_{2}=\sqrt{\int \hat{x} \cdot \hat{x} d x} \\
\sigma(k, t) & =\frac{d \ln (a(k, t))}{d t}
\end{aligned}
$$

where $k$ is the wave number and assuming that the growth rate is constant between two derivation steps. The perturbative components are computed by subtracting the components from the non-linear perturbed solution to the components from the unperturbed base state. No finger emerge in this latter case.

First, we compare in Fig. 6 the results obtained from the gray lattice Boltzmann and the Brinkman force models. Both approaches lead to equivalent growth rates. Thus, at early times, the linearly development of the instability is well 
captured by the two models. The growth rate decreases in time. The most dangerous wave number corresponding to the largest growth rate as well as the threshold and the cutoff wave numbers are reduced as the instability progresses resulting in widening fingers.

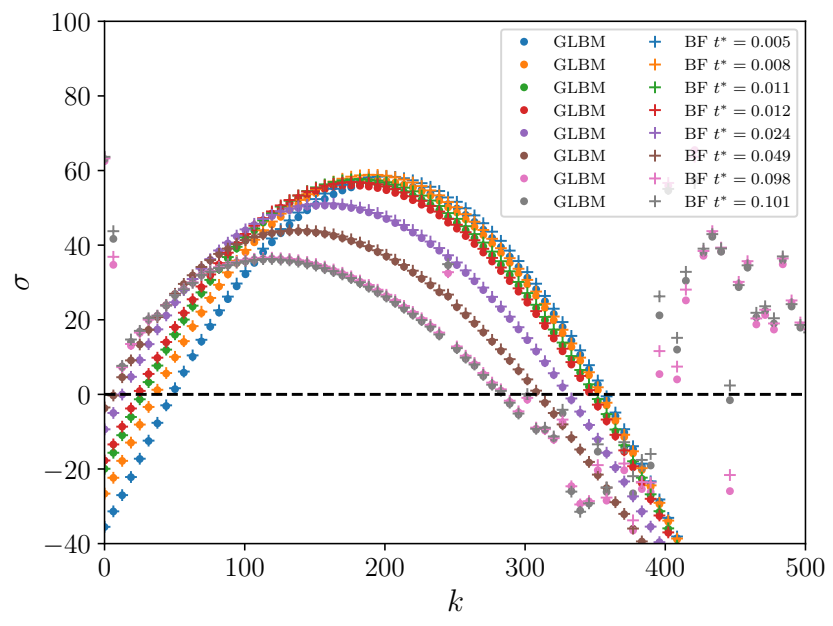

Fig. 6 Dispersion curves for $R=3, P_{e}=2000$ at various times. - gray lattice Boltzmann, + Brinkman force model.

Diffusion acts as a stabilization factor by smoothing perturbations and heading to an homogeneous mixture. Hence, as shown in Fig. 7, the instability is more dominant with higher Péclet numbers. A lower fluid dispersion expands the range of unstable wave numbers and increases the growth rate. The cutoff wave number increases with the Péclet number whereas it has a very limited influence on the threshold wave number. These results are in qualitative agreement with the growth rates obtained by linear stability in Ref. [8]. Remark that the choice of scaling is not neutral for the linear stability analysis. As noted by the previous authors, taking the reference length and time as the diffusive length $l_{\text {ref }}=\mathcal{D} / U$ and $t_{\text {ref }}=L_{r e f} / U$ leads to dispersion equations independent of the Péclet number in the linear stability analysis [7] whereas in the case of the convective length $l_{r e f}=n_{y}$ and $t_{r e f}=L_{r e f} / U$, dispersion curves depends on the Péclet number [8]. The Péclet number also influences the transition from linear to non-linear instability. At $t^{*}=0.1$, for $P_{e}=5000$, fingers starts to interact non-linearly whereas for lower Péclet numbers, the perturbation is still well-described by Eq. 26. The present non-linear simulations confirm the important role of the Péclet number in the development of the instability at early times.
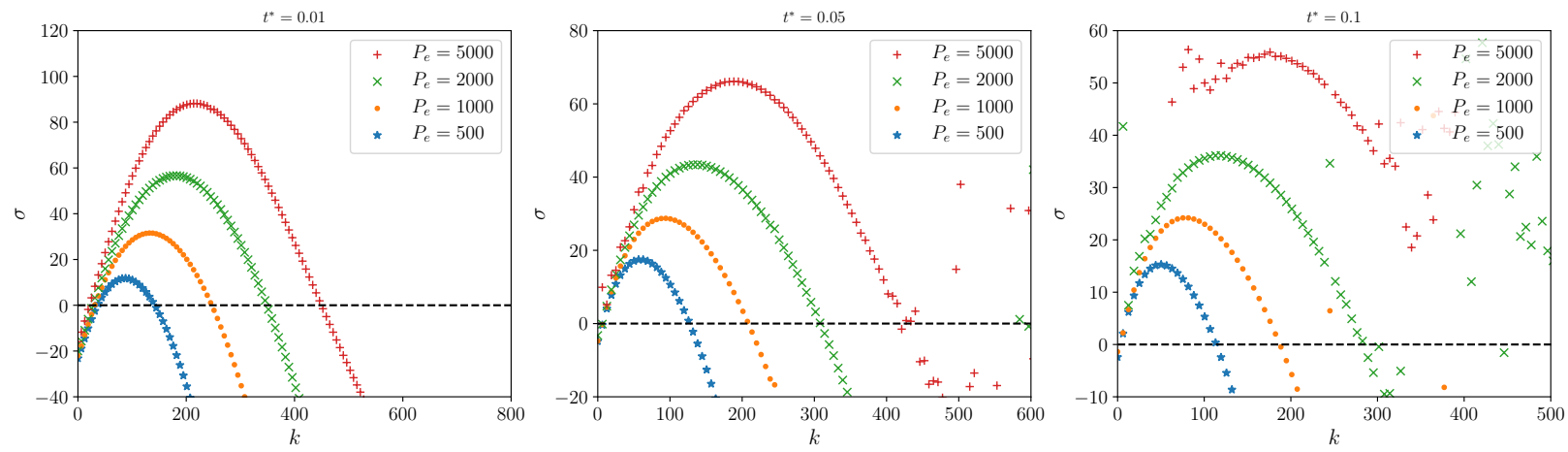

Fig. 7 Dispersion curves for $R=3$ at $t^{*}=0.01,0.05,0.1$ with different Péclet values. Simulations are performed with the gray lattice Boltzmann model.

As observed in Fig. (5), the value of the effective viscosity changes the beginning of the instability. The linear growth of the fingers is also affected. At early times, the dissipation due to the effective viscosity reduces the growth rate of the perturbation and the cutoff wave number also decreases as shown in Fig. 8. However, the time evolution of 
the growth rate and the cutoff wave number is different from the effect of the diffusion caused by a low Péclet number. The growth rate for $\mu_{e, m}=10 \mu_{m}$ increases slightly from $t^{*}=0.01$ to $t^{*}=0.05$ and then keeps constant from $t^{*}=0.05$ to $t^{*}=0.1$. The cutoff wave number stays at $k \approx 200$. For $\mu_{e, m}=0.1 \mu_{m}$ and $\mu_{e, m}=\mu_{m}$, the growth rate and the cutoff wave number decrease in time. As the instability progresses, the differences resulting from the effective viscosity are less visible.
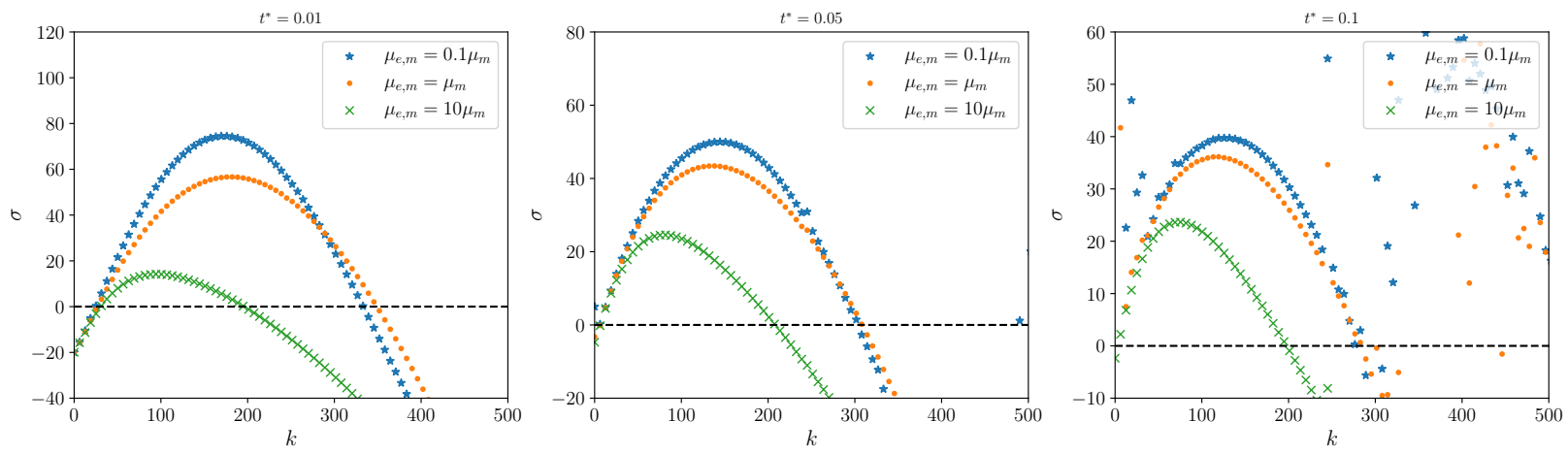

Fig. 8 Dispersion curves for $R=3, P_{e}=2000$ at $t^{*}=0.01,0.05,0.1$ with different effective viscosity values. Simulations are performed with the gray lattice Boltzmann model.

\section{Intermediate times: non-linear interactions}

At early times, the sharp interface diffuses and begins to deform. This stage is called the linearly unstable regime and linear stability analysis tools are suitable to describe the instability. The fingers then start to interact via various nonlinear mechanisms. Many thin fingers develop, and strong linear interactions take place, which lead globally to a coarsening of the fingers in the transverse direction and a growth of the fingers in the streamwise direction. The number of fingers and the intensity of these interactions increase with the Péclet number and the log-viscosity ratio. The influence of the effective viscosity is less significant as long as the interface is not overly diffuse after the delay in the emergence of fingers. The interactions are also dependent of the noise of the initial perturbation and may vary from simulation to simulation. Results obtained from the Gray lattice Boltzmann model and the Brinkman force model are identical if the same initial perturbation is applied.

Figure 9 shows a sequence of snapshots of a simulation performed with the Brinkman force model at $R=3$, $P_{e}=16000$. This high Péclet number is chosen to assure intense interactions between fingers for better identification of the main non-linear mechanisms. These include spreading, shielding, fading, and coalescence. By spreading, we refer to the process whereby one finger is slightly ahead of others. The finger grows very quick and the gradient steepens between the finger and the surrounding more viscous fluid. The finger may then widens at the tip and shields the growth of the smaller neighbouring fingers. Few thin fingers fade and diffuse in the ambient fluid resulting in a region of intermediate viscosity. Coalescence phenomenon describes the merging of two or more fingers together. The present dynamics of the simulation is rich displaying all major mechanisms, and exhibits also more complex behaviors such as tip splitting, when a finger splits into two at the tip and side branching when a finger splits at its side. These fascinating interaction mechanisms occurs in the nonlinear regime.

\section{Conclusion}

In this study, the lattice Boltzmann simulation of the viscous fingering instability is discussed. The global dynamic is presented, and we especially focus on two stages of the development of the instability. First, the very beginning of the instability, when the fingers grow linearly, is studied by computing the growth rate associated to the perturbation. Secondly, we describe qualitatively intermediate times when strong non-linear interactions take place.

For these two stages, we show that both of the gray lattice Boltzmann and Brinkman force porous models give the same result despite of being based on different formulations. Both result in Darcy-Brinkman equations that is why the influence of the viscous dissipation on the instability is then explored by varying the value of the effective viscosity. A high value of the effective viscosity causes a delay in the emergence of fingers whereas a low value of the effective viscosity has not a significant effect on the instability. Moreover, the differences are reduced when the instability is 


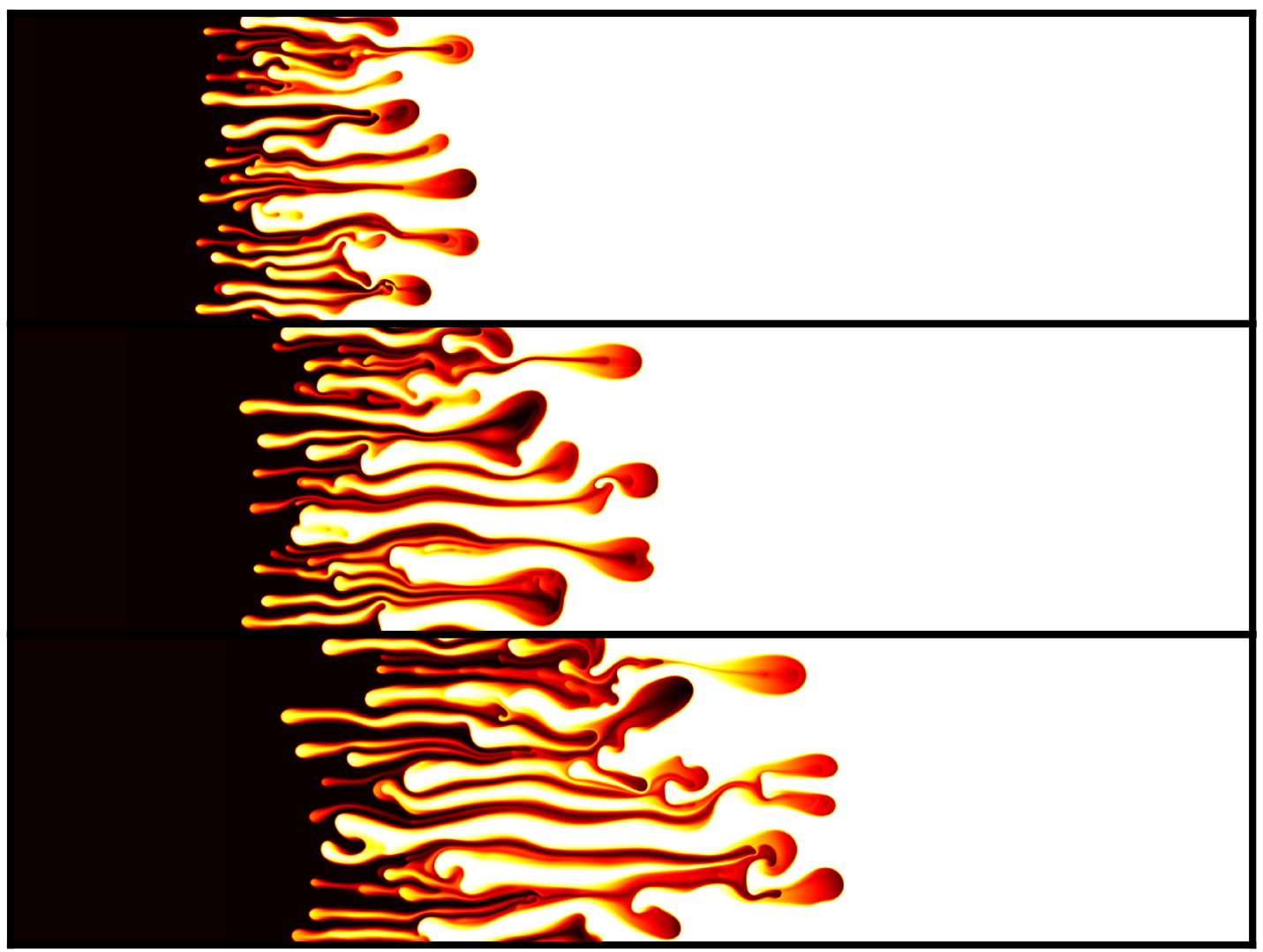

Fig. 9 Instability development for $R=3, P_{e}=16000$ at $t^{*} \approx 0.91,1.22,1.52$. The whole domain $\left(n_{x}=\right.$ 4096, $\left.n_{y}=1024\right)$ is plotted. The color-map (black-red-yellow-white) represents the variation of the molar fraction from $x_{1}=0.9$ to $x_{1}=0.1$. Same scaling is used for both $\mathbf{x}$ and $\mathbf{y}$-axis.

growing up. Indeed, contrary to the Darcy drag, the terms related to the effective viscosity are not the driving force of the instability. We also observed that a high Péclet number leads to a more intense instability. In the linear regime, the growth rate as well as the most dangerous and the cutoff numbers increase with the Péclet number. In addition, more fine-scale fingers develop and interact considerably in the non-linear regime at high Péclet number.

\section{Appendix}

\section{A. Macroscopic limit of the Lattice Boltzmann model for miscible mixtures}

The macroscopic limit of a lattice Boltzmann scheme can be obtained via the Chapman-Enskog analysis. This multiple scale expansion provides a relation between the mesoscopic scale of the Boltzmann equation and the macroscopic scale of the Navier-Stokes equation. This derivation is straightforward and is similar to the standard lattice Boltzmann model with a force arising from Guo's forcing scheme (see Ref. [5]). Therefore in the low Mach and continuum limit, the kinetic equation Eq. (1) and its moments Eq. (6) are equivalent to the following hydrodynamics equations,

$$
\partial_{t} \rho_{m}+\nabla \cdot\left(\rho_{m} \mathbf{u}_{m}\right)=0,
$$




$$
\partial_{t}\left(\rho_{m} \mathbf{u}_{m}\right)+\nabla \cdot\left(\rho_{m} \mathbf{u}_{m} \otimes \mathbf{u}_{m}\right)=-\nabla p_{m}+\nabla \cdot\left[\mu_{m}\left(\nabla \mathbf{u}_{m}+\left(\nabla \mathbf{u}_{m}\right)^{T}\right)\right]-p \sum_{n=1}^{N} \frac{x_{m} x_{n}}{\mathcal{D}_{m n}}\left(\mathbf{u}_{m}-\mathbf{u}_{n}\right)
$$

where the partial pressure is equal to $p_{m}=\rho_{m} c_{s, m}^{2}$, and the total pressure is given by Dalton's law $p=\sum_{m=1}^{N} p_{m}$. The dynamic viscosity of species $m$ is expressed in terms of the relaxation time according to

$$
\mu_{m}=\rho_{m} c_{s}^{2}\left(\tau_{m}-\frac{\delta_{t}}{2}\right) .
$$

\section{Acknowledgments}

This work was performed using HPC resources from GENCI-CINES (Grant 2018-A0052A10636)

\section{References}

[1] Homsy, G. M., "Viscous Fingering in Porous Media," Annual Review of Fluid Mechanics, Vol. 19, No. 1, 1987, pp. $271-311$. doi:10.1146/annurev.fl.19.010187.001415, URL https://doi.org/10.1146/annurev.fl.19.010187.001415

[2] Hill, S., and P, F., "Channeling in packed columns," Chemical Engineering Science, Vol. 1, No. 6, 1952, pp. $247-253$. doi:10.1016/0009-2509(52)87017-4, URL https://doi .org/10.1016/0009-2509(52)87017-4

[3] Saffman, P. G., and Taylor, G. I., "The penetration of a fluid into a porous medium or Hele-Shaw cell containing a more viscous liquid," Proceedings of the Royal Society of London. Series A. Mathematical and Physical Sciences, Vol. 245, No. 1242, 1958, pp. 312-329. doi:10.1098/rspa.1958.0085, URL https://doi.org/10.1098/rspa.1958.0085

[4] Vienne, L., Marié, S., and Grasso, F., "Lattice Boltzmann model for miscible gases: a forcing term approach,” submitted, 2019.

[5] Guo, Z., Zheng, C., and Shi, B., "Discrete lattice effects on the forcing term in the lattice Boltzmann method," Phys. Rev. E, Vol. 65, 2002, p. 046308. doi:10.1103/PhysRevE.65.046308, URL https://link.aps.org/doi/10.1103/PhysRevE.65.046308

[6] Kerkhof, P. J. A. M., and Geboers, M. A. M., “Toward a unified theory of isotropic molecular transport phenomena,” AIChE Journal, Vol. 51, No. 1, 2004, pp. 79-121. doi:10.1002/aic.10309, URL/https://doi.org/10.1002/aic.10309

[7] Tan, C. T., and Homsy, G. M., "Stability of miscible displacements in porous media: Rectilinear flow," Physics of Fluids, Vol. 29, No. 11, 1986, p. 3549. doi:10.1063/1.865832, URL https://doi.org/10.1063/1.865832

[8] Pramanik, S., and Mishra, M., "Effect of Péclet number on miscible rectilinear displacement in a Hele-Shaw cell," Physical Review E, Vol. 91, No. 3, 2015. doi:10.1103/physreve.91.033006, URL https://doi.org/10.1103/physreve.91.033006

[9] Hota, T. K., Pramanik, S., and Mishra, M., "Nonmodal linear stability analysis of miscible viscous fingering in porous media," Physical Review E, Vol. 92, No. 5, 2015. doi:10.1103/physreve.92.053007, URL https://doi.org/10.1103/physreve. 92.053007

[10] Nijjer, J. S., Hewitt, D. R., and Neufeld, J. A., “The dynamics of miscible viscous fingering from onset to shutdown," Journal of Fluid Mechanics, Vol. 837, 2018, pp. 520-545. doi:10.1017/jfm.2017.829, URL https://doi.org/10.1017/jfm.2017. 829 .

[11] Norouzi, M., and Shoghi, M. R., "A numerical study on miscible viscous fingering instability in anisotropic porous media," Physics of Fluids, Vol. 26, No. 8, 2014, p. 084102. doi:10.1063/1.4891228, URL https://doi.org/10.1063/1.4891228

[12] Walsh, S. D., Burwinkle, H., and Saar, M. O., "A new partial-bounceback lattice-Boltzmann method for fluid flow through heterogeneous media," Computers \& Geosciences, Vol. 35, No. 6, 2009, pp. 1186-1193. doi:10.1016/j.cageo.2008.05.004, URL https://doi.org/10.1016/j.cageo.2008.05.004.

[13] Zhu, J., and Ma, J., "An improved gray lattice Boltzmann model for simulating fluid flow in multi-scale porous media," Advances in Water Resources, Vol. 56, 2013, pp. 61-76. doi:10.1016/j.advwatres.2013.03.001, URL https://doi.org/10.1016/j. advwatres.2013.03.001

[14] Yoshida, H., and Hayashi, H., "Transmission-Reflection Coefficient in the Lattice Boltzmann Method," Journal of Statistical Physics, Vol. 155, No. 2, 2014, pp. 277-299. doi:10.1007/s10955-014-0953-7, URL https://doi.org/10.1007/s10955014-0953-7. 
[15] Thorne, D., and Sukop, M., "Lattice Boltzmann model for the elder problem," Computational Methods in Water Resources: Volume 2, Proceedings of the XVth International Conference on Computational Methods in Water Resources, Elsevier, 2004, pp. 1549-1557. doi:10.1016/s0167-5648(04)80165-5, URL https://doi .org/10.1016/s0167-5648(04)80165-5.

[16] Chen, C., Li, L., Mei, R., and Klausner, J. F., "Chapman-Enskog Analyses on the Gray Lattice Boltzmann Equation Method for Fluid Flow in Porous Media," Journal of Statistical Physics, Vol. 171, No. 3, 2018, pp. 493-520. doi:10.1007/s10955-018-2005-1, URL https://doi.org/10.1007/s10955-018-2005-1

[17] Krüger, T., Kusumaatmaja, H., Kuzmin, A., Shardt, O., Silva, G., and Viggen, E. M., The Lattice Boltzmann Method, Springer International Publishing, 2017. doi:10.1007/978-3-319-44649-3, URL https://doi .org/10.1007/978-3-319-44649-3

[18] Ginzburg, I., "Consistent lattice Boltzmann schemes for the Brinkman model of porous flow and infinite Chapman-Enskog expansion,” Physical Review E, Vol. 77, No. 6, 2008. doi:10.1103/physreve.77.066704, URL https://doi.org/10.1103/ physreve.77.066704

[19] Guo, Z., and Zhao, T. S., "Lattice Boltzmann model for incompressible flows through porous media," Physical Review E, Vol. 66, No. 3, 2002. doi:10.1103/physreve.66.036304, URL https://doi .org/10.1103/physreve.66.036304

[20] Martys, N. S., "Improved approximation of the Brinkman equation using a lattice Boltzmann method," Physics of Fluids, Vol. 13, No. 6, 2001, pp. 1807-1810. doi:10.1063/1.1368846, URL https://doi.org/10.1063/1.1368846

[21] Spaid, M. A. A., and Phelan, F. R., "Lattice Boltzmann methods for modeling microscale flow in fibrous porous media," Physics of Fluids, Vol. 9, No. 9, 1997, pp. 2468-2474. doi:10.1063/1.869392, URL https://doi .org/10.1063/1.869392

[22] Grosfils, P., Boon, J. P., Chin, J., and Boek, E. S., "Structural and dynamical characterization of Hele-Shaw viscous fingering," Philosophical Transactions of the Royal Society of London. Series A: Mathematical, Physical and Engineering Sciences, Vol. 362, No. 1821, 2004, pp. 1723-1734. doi:10.1098/rsta.2004.1398, URL/https://doi.org/10.1098/rsta.2004.1398

[23] Talon, L., Bauer, D., Gland, N., Youssef, S., Auradou, H., and Ginzburg, I., "Assessment of the two relaxation time Lattice-Boltzmann scheme to simulate Stokes flow in porous media," Water Resources Research, Vol. 48, No. 4, 2012. doi:10.1029/2011wr011385, URL https://doi.org/10.1029/2011wr011385.

[24] Khirevich, S., Ginzburg, I., and Tallarek, U., "Coarse- and fine-grid numerical behavior of MRT/TRT lattice-Boltzmann schemes in regular and random sphere packings," Journal of Computational Physics, Vol. 281, 2015, pp. 708-742. doi: 10.1016/j.jcp.2014.10.038, URL https://doi.org/10.1016/j.jcp.2014.10.038

[25] Ginzburg, I., "Comment on An improved gray Lattice Boltzmann model for simulating fluid flow in multi-scale porous media: Intrinsic links between LBE Brinkman schemes," Advances in Water Resources, Vol. 88, 2016, pp. $241-249$. doi:10.1016/j.advwatres.2014.05.007, URL https://doi.org/10.1016/j .advwatres.2014.05.007.

[26] Coveney, P. V., Succi, S., d'Humières, D., Ginzburg, I., Krafczyk, M., Lallemand, P., and Luo, L.-S., "Multiple\&\#x2013;relaxation\&\#x2013;time lattice Boltzmann models in three dimensions," Philosophical Transactions of the Royal Society of London. Series A: Mathematical, Physical and Engineering Sciences, Vol. 360, No. 1792, 2002, pp. 437-451. doi:10.1098/rsta.2001.0955.

[27] Zou, Q., and He, X., "On pressure and velocity boundary conditions for the lattice Boltzmann BGK model," Physics of Fluids, Vol. 9, No. 6, 1997, pp. 1591-1598. doi:10.1063/1.869307, URL https://doi.org/10.1063/1.869307

[28] Vergnault, E., Malaspinas, O., and Sagaut, P., "A lattice Boltzmann method for nonlinear disturbances around an arbitrary base flow," Journal of Computational Physics, Vol. 231, No. 24, 2012, pp. 8070-8082. doi:10.1016/j.jcp.2012.07.021, URL https://doi.org/10.1016/j.jcp.2012.07.021.

[29] Pérez, J. M., Aguilar, A., and Theofilis, V., "Lattice Boltzmann methods for global linear instability analysis," Theoretical and Computational Fluid Dynamics, Vol. 31, No. 5-6, 2016, pp. 643-664. doi:10.1007/s00162-016-0416-7, URL https: //doi.org/10.1007/s00162-016-0416-7. 